Articles, an editorial and a comment in this number reflect on the historical and current importance of clinical audit in evaluating a variety of aspects of health services. From France, Italy, The Netherlands, Norway, and the United
Kingdom come reflections upon specific services and on whole national scenarios, employing a variety of epidemiological approaches to the problems.

\title{
Editorial
}

\section{Medical audit in The Netherlands: experience over 22 years}

The first medical audit in The Netherlands was undertaken in 1973 in a general hospital. At the same, the National Medical Specialist Organisation prepared a policy paper on the introduction of medical audit, or peer review as it was called, in all Dutch hospitals on a voluntary basis. The approach to medical audit proposed in that paper was based on experience gained in the United States. It is problem oriented-not physician or patient oriented-and consists of the following steps:

- Choosing a topic,

- Setting criteria,

- Gathering data (generally retrospectively),

- Comparing criteria with actual practice and, when necessary,

- Introducing changes in health care provision.

In this editorial the term "medical audit" will be used in its internationally accepted sense as quality assurance by medical professionals. A second policy paper, published in 1976 in cooperation with the Medical Association of Hospital Directors, advised the setting up of a National Organisation for Peer Review in Hospitals (CBO) to support and advise hospital staff on the implementation of medical audit. ${ }^{1}$ The CBO is funded by the hospitals - each pays the organisation a small percentage of the cost of each inpatient day. The fact that Dutch hospitals were seen to have well organised and well functioning medical staff seemed to be a good basis for the implementation of medical audit. The position of the CBO was partly influenced by external pressure from the Dutch government which had just put forward a bill proposing external quality assessment of medical specialist care. This law was never passed, but the introduction of medical audit can be considered as self regulation by the profession to maintain its autonomy, a situation quite different from the introduction of medical audit in the UK. ${ }^{2}$

In the intervening years there has been no formal evaluation of medical audit on a national or an institutional level. This seems to be the general case with regard to quality assurance programmes. ${ }^{3}$ A written survey in 1984 , which covered about $50 \%$ of Dutch hospitals, showed that the majority of medical staffs had established a medical audit committee but that most of these were dormant. ${ }^{4}$ After studying the published report, and after discussions with some key people in this area, we concluded that most hospitals had applied medical audit at some time, but very few had continued with this over the years. ${ }^{2}$ Medical audit was considered by most clinicians to be a long term, time consuming task and the problems encountered with gathering data could not yet be balanced against any clear improvement in patient care. Nevertheless, there is some useful feedback after 22 years of medical audit.

Firstly, the topics that were chosen for audit studies give some insight into the potential problems with quality that clinicians encounter in health care provision. The topics give an impression of the epidemiology of (bad) quality. In most hospitals, topics are chosen once a year by a team of medical specialists, based on a structure described by Williamson. ${ }^{5}$ Over the years, similar types of quality problems have continued to arise ${ }^{67}$ Most are medical problems involving aspects such as blood transfusion, preoperative anticoagulant therapy, and monitoring of nephrotoxic antibiotics, but issues related to the organisation of care and communication between health care providers, such as deficient recording of medical records are also included. It is noteworthy that no topics on the more behavioural aspects of medical care have been mentioned. This can be explained, in part, by the inherent problems in setting criteria and gathering data when considering these topics. The table gives an overview of the 12 topics that were selected most frequently as numbers 1,2 , and 3 during 101 priority setting meetings in 41 hospitals over the years 1977-92. ${ }^{7}$ As can be seen, most topics concern the process of care-patient outcome criteria were seldom addressed.

The second point worthy of mention is the results reported from local medical audit studies. Findings of some studies were published, especially in the early years, both in the Dutch and international papers and in the CBO newsletter. For example, the positive effects of medical audit have been described with regard to: blood transfusion therapy, ${ }^{8,9}$ information to general practitioners after patient discharge from hospital, ${ }^{10}$ information given to orthognatic

The 12 topics selected most frequently as priorities number 1, 2, or 3 during 101 priority meetings in 41 hospitals between 1977 and 1992.*

\begin{tabular}{lll}
\hline Topic & & No of times mentioned \\
\hline 1 & Preoperative assessment & 30 \\
2 & Medical record keeping & 20 \\
3 & Anticoagulant use & 16 \\
4 & Antibiotics policy & 15 \\
5 & Blood transfusion policy & 15 \\
6 & Reanimation policy & 12 \\
7 & Bladder catheterisation & 10 \\
8 & Triage at the emergency room & 9 \\
9 & Inter-colleague consultation & 8 \\
10 & Infusion policy & 7 \\
11 & Urgent laboratory tests & 7 \\
12 & Bedsores policy & 7 \\
\hline
\end{tabular}

* Adapted from Klazinga (7). 